\title{
Breathlessness in a patient with rheumatoid vasculitis
}

\author{
Neslihan Başçll, Tanju Tütüncü, Gül Kısacık, Ayşen Sivri, Lütfi Çöplü
}

A 48-year-old man was admitted to our out-patient clinic with a 3-month history of progressive dyspnoea. He had had rheumatoid arthritis for 30 years. According to the criteria of the American College of Rheumatology, he had classical rheumatoid arthritis with negative rheumatoid factor in the serum. Due to osteoporosis, bone and cartilage destruction, his functional capacity was evaluated as class 4 . His case history revealed that his joint involvement had progressed despite several courses of salicylates, indomethacin, hydroxychloroquine and steroids. Azathioprine had been added to his medication 4 months earlier, due to swelling of the knee and the interphalangeal joints. Although there was symptomatic improvement, his exercise tolerance had deteriorated.

Physical examination revealed central cyanosis with respiratory distress. His temperature was $37.8^{\circ} \mathrm{C}$, blood pressure $160 / 80 \mathrm{mmHg}$, pulse rate 118 beats/min and respiration rate 26 breaths/min. Hepatojugular reflux and hepatomegaly were noticed. The pulmonary component of the second heart sound was accentuated. His hands and feet had the classic advanced deformities of rheumatoid arthritis including a swan neck deformity of the fingers. Chest $\mathrm{X}$-ray showed an increase in the size of the cardiac silhouette when compared with one taken 3 months earlier (figure 1). Pulmonary conus was prominent and the lung fields were clear with increased hyperlucency. Electrocardiography provided evidence of pulmonary hypertension with P-pulmonale and right ventricular hypertrophy. Echocardiography also showed dilatation of the right ventricle with a pulmonary arterial pressure of $70 \mathrm{mmHg}$ and minimal pericardial effusion.

He was hospitalised and investigated for the underlying pathology. Venous Doppler monitoring of the lower extremities and abdomen revealed a patent venous system with no deep venous thrombosis. Ventilation-perfusion scanning of the lungs showed no evidence of thromboembolic phenomenon. Pulmonary angiography demonstrated the dilated main pulmonary artery with its right and left main branches, and a perfusion defect at the periphery of both lung fields symmetrically (figure 2). High-resolution computed tomography (CT) of the thorax revealed pulmonary arterial dilatation with no evidence of emphysema, or pleural or parenchymal lung disease (figure 3).

Laboratory tests showed negative rheumatoid factor, antiphospholipid antibodies and lupusanticoagulant. Antinuclear antibody and anti-DNA antibody were also negative. His erythrocyte sedimentation rate was $48 \mathrm{~mm} / \mathrm{h}$ and a complete blood count was within normal limits. Arterial blood gas analysis revealed mild respiratory alkalosis with $\mathrm{pH} 7.46, \mathrm{pCO}_{2} 29.5$ $\mathrm{mmHg}, \mathrm{pO}_{2} 60 \mathrm{mmHg}$ and $\mathrm{HCO}_{3} 28 \mathrm{mEq} / \mathrm{l}$. Pulmonary function tests including spirometry, lung volume and diffusion tests were all within normal limits: forced vital capacity (FVC):

Faculty of Medicine, Hacettepe University, Ankara, Turkey Department of Internal Medicine N Baş̧̨l

Department of General Surgery

T Tütüncü

Department of Chest

Disease

G Kısacik

L Çöplü

Department of

Physiotherapy and

Rehabilitation

A Sivri

Correspondence to Neslihan Basçl, MD, Sancak Mahallesi, 221 Sokak No

5/10, 06550 Yildiz, Ankara, Turkey

Accepted 16 December 1997
4.961 (133\% of predicted), forced expiratory volume in $1 \mathrm{~s}\left(\mathrm{FEV}_{1}\right): 3.651(119 \%$ of predicted), $\mathrm{FEV}_{1} / \mathrm{FVC}=90 \%$, total lung capacity (TLC) $108 \%$, residual volume (RV) $116 \%$, diffusing capacity of CO (DLCO): 7.2 $\mathrm{mmol} / \mathrm{kPa} \cdot \mathrm{min}(99 \%$ of predicted), DLCO/ pulmonary capillary blood volume: 1.00 , DLCO/1 (82\% of predicted).

The patient refused thoracotomy, which had been planned to get a specimen for histologic examination. $\mathrm{He}$ was digitalised and antihypertensive medication was started. The patient was treated appropriately after ruling out possible disease states. Although he improved in the following 20 weeks, the disease seemed to progress in the last few weeks despite treatment and he died of severe respiratory failure. No post-mortem was held because of the religious beliefs of the family.

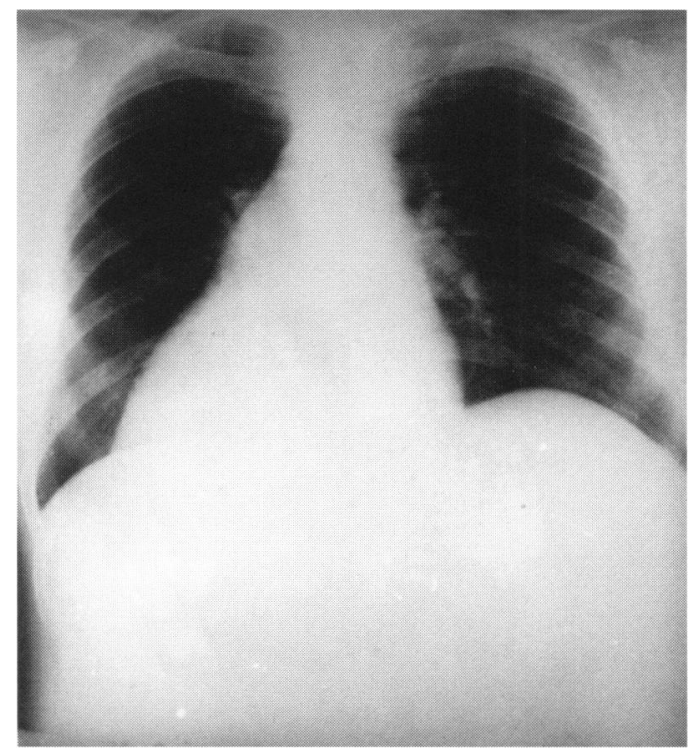

Figure 1 Chest $\mathrm{X}$-ray showing cardiac silhouette enlargement 


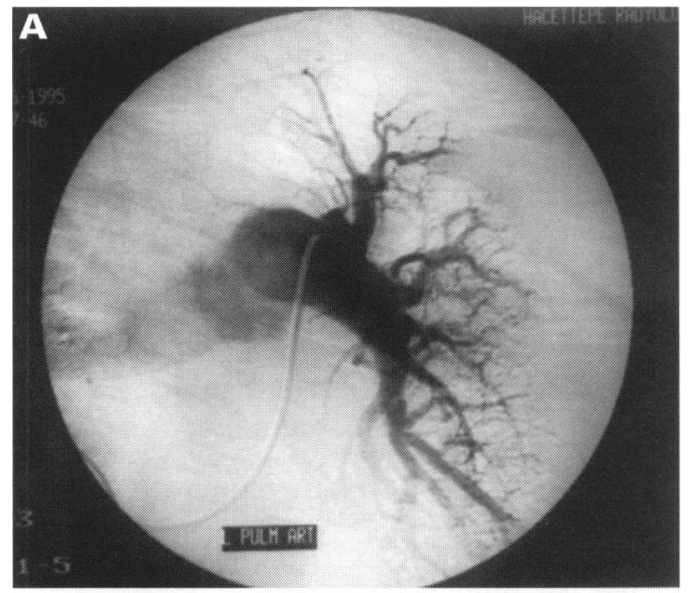

Figure 2 Pulmonary angiography demonstrating (A) the dilated main pulmonary artery, and (B) the symmetrical perfusion defect

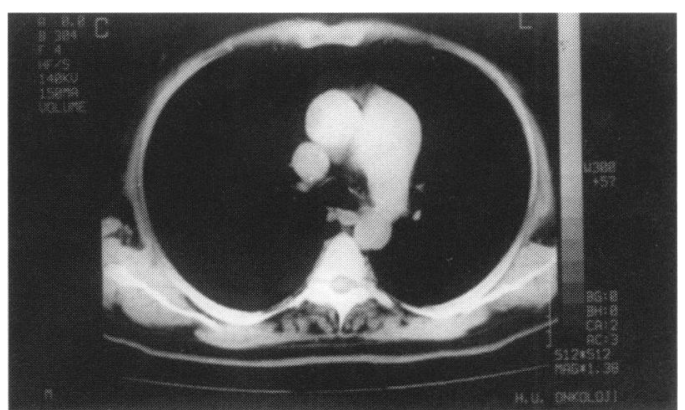

Figure 3 Thoracic CT scan

\section{Questions}

1 What is the most probable diagnosis?

2 Discuss the differential diagnosis.

3 What are the treatment options for this patient ? 


\section{Answers}

QUESTION 1

A 3-month history of progressively increasing dyspnoea with central cyanosis, tachycardia, tachypnoea, positive hepatojugular reflux and hepatomegaly make the diagnosis of respiratory distress with right heart failure that is cor pulmonale. The laboratory evidence of cardiomegaly with prominence of pulmonary conus on chest X-ray, electrographic findings of right ventricular hypertrophy, and echocardiographic evidence of pulmonary hypertension confirm the diagnosis.

\section{QUESTION 2}

Differential diagnosis of acute cor pulmonale includes the parenchymal and pleural diseases of the lung, cardiac pathologies, pulmonary thromboembolic events and pulmonary vascular diseases. Other causes of the cor pulmonale were excluded on the basis of the chest CT scan, venous Doppler monitoring of the lower and abdominal venous system, and echocardiography. Primary pulmonary vascular disease was therefore considered. Although pulmonary vasculitis in association with rheumatoid arthritis is rare, it cannot be ruled out in such a patient with no other possible underlying cause of pulmonary hypertension.

\section{QUESTION 3}

The treatment of vasculitis involves immunosuppressive regimens including steroids, cyclophosphamide, and azathiopurine. There are studies showing the beneficial effects of the analogues of endothelial nitric oxide synthetase in patients with pulmonary hypertension. Intravenous prostacycline can also reduce pulmonary arterial pressure. While treating the underlying cause of the pulmonary hypertension, supportive care with proper oxygenation, digitalisation, and treatment with arterial vasodilators should not be forgotten.

\section{Discussion}

Pulmonary arteritis is a rare complication of rheumatoid arthritis. ${ }^{1}$ Although vasculitis could not be demonstrated by biopsy, pulmonary hypertension due to pulmonary vasculitis was considered because other diseases that could lead to pulmonary hypertension in a patient with rheumatoid arthritis had been excluded. Systemic vasculitis usually accompanies pulmonary hypertension. ${ }^{1}$ In our case there was no suggestion of systemic vasculitis, ie, no cutaneous ulceration, dermal necrosis, polyneuropathy or mononeuritis multiplex, prior to his presentation with breathlessness or during 5 months follow-up.

When a patient with rheumatoid arthritis complains of breathlessness, pulmonary vascular disease and small airway obstruction should be considered in the differential diagnosis. Vasculitis in rheumatoid arthritis, as in other collagen vascular diseases, involves small arteries and arterioles. In our case the pulmonary angiogram demonstrating the symmetrically nonperfused areas at the periphery of both lung fields strongly suggests the involvement of the small arteries and arterioles which normally perfuse the peripheral regions of the lung.

Factors predicting life expectancy in pulmonary vasculitis have not yet been well defined. Right heart failure during the course of disease is associated with poor outcome. Radiographic evidence of cardiac enlargement and evidence of right heart strain on electrocardiogram at presentation is also predictive of survival for less than 5 years. There is no relation between pulmonary arterial pressure and length of survival. ${ }^{2}$ Our patient had right heart failure at presentation.

Very few cases of pulmonary hypertension secondary to rheumatoid vasculitis have been documented. To our knowledge, only five other cases of rheumatoid pulmonary vasculitis without underlying parenchymal lung disease have been reported since $1965 . .^{134}$ Of these, there are only three cases, including ours, in which the pulmonary vessels were the only target of rheumatoid vasculitis. Differential diagnosis of pulmonary hypertension may be difficult even if lung biopsy has been performed. Distinction between vasculitis and changes secondary to pulmonary hypertension of any cause can be difficult.

The diagnosis of pulmonary hypertension is reached by exclusion. ${ }^{15}$ The age and sex of our patient were unusual for primary pulmonary hypertension. The diagnosis of diffuse pulmonary fibrosis is ordinarily based upon the radiographic appearance of the lung fields and if routine X-rays of the chest fail to reveal even severe pulmonary fibrosis, then pulmonary function tests may be of great value in differentiating between pulmonary fibrosis and pulmonary vascular disease. Pulmonary function studies in patients with pulmonary hypertension secondary to interstitial fibrosis reveal reduced lung volumes and the carbon monoxide diffusing capacity, measured by the single breath method, is one third or less of predicted values. ${ }^{135}$ In this case, these tests were within normal limits. In patients with pulmonary hypertension secondary to primary vascular disease, the lung volumes are generally normal and the diffusing capacity averages $55 \%$ of predicted values. ${ }^{5}$ It is well known that collagen vascular diseases are associated with vasculitis, but pulmonary vasculitis in association with rheumatoid arthritis is rare. The rheumatoid disease may be mild, as judged by articular involvement and clinicians should be suspicious of breathlessness in patients with rheumatoid arthritis but no evidence of pleural or interstitial lung disease.

It is possible that earlier diagnosis and immunosuppressive treatment of the vasculitis might delay or even prevent a fatal outcome. The development of pulmonary vasculitis is too unusual to recommend routine surveillance in all patients with rheumatoid arthritis, but it is suggested that early physiological investigation of dyspnoeic symptoms might indicate which patients need screening for pulmonary hypertension. 


\section{Final diagnosis}

Acute cor pulmonale and pulmonary vasculitis.

1 Balagopal VP, Costa P, Greenstone MA. Fatal pulmonary hypertension and rheumatoid vasculitis. Eur Respir $\mathcal{f}$ 1995;8:331-3.

2 Rozkovec P, Montanes P, Oakley CM. Factors that influence the outcome of primary pulmonary hypertension. Br Heart $\mathcal{F}$ 1986;55:449-58.

3 Baydur A, Mongan ES, Slager UT. Acute respiratory failure and pulmonary arteritis without parenchymal involvement. Chest 1979;75:518-20.
Keywords: pulmonary hypertension; rheumatoid arthritis; pulmonary vasculitis; cor pulmonale; breathlessness

4 Kay JM, Banik S. Unexplained pulmonary hypertension with pulmonary arteritis in rheumatoid disease. $B r f D i s$ Chest 1977;71:53-9.

5 Williams WH, Adler JJ, Colp C. Pulmonary function studies as an aid in the differential diagnosis of pulmonary hypertension. $A m \mathcal{F}$ Med 1969;47:378-83.

\title{
Knee pain - a false lead
}

\author{
Rosemary Morgan
}

A 78-year-old man was admitted to hospital via his general practitioner with a three-day history of severe pain in his left knee and poor mobility. He lived alone and until this admission had been independently mobile and self-caring. He was cognitively intact. There was no history of falls or trauma. He had a history of gout; his last flare up had been more than two years ago and he had been taking allopurinol on a regular basis since. Acute gout had previously affected his big toe (first metatarsophalangeal joint) on the right foot but no other joints had been affected.

On examination he looked well but was in pain and apyrexial. Examination of his left knee revealed it to be exquisitely tender with a small effusion. It was not red or hot compared to the right knee which also had a small effusion. Inspection revealed both limbs to be equal in length. An X-ray of his left knee showed osteoarthritis of the joint but nothing else. His initial full blood count, serum urea and creatinine, random blood glucose, rheumatoid factor, C-reactive protein and erythrocyte sedimentation rate were all within the normal range. Blood cultures were negative. Aspiration of the left knee yielded $6 \mathrm{ml}$ of clear straw-coloured fluid only, both Gram stain and polarised light microscopy were reported as negative. His serum uric acid level was 0.48 $\mathrm{mmol} / \mathrm{l}$. He was commenced on regular indomethacin and cold compresses were applied to his left knee, a provisional diagnosis of osteoarthritis being made. Twenty-four hours after admission, despite regular analgesia, he was still complaining of pain and was given a corticosteroid injection into the left knee. Tests for prostatic specific antigen and liver function tests were normal. On further inquiry he still denied any history of trauma but recollected that three days prior to admission he had turned suddenly whilst getting food out of his fridge and his painful left knee had developed from that time. On day 3 of his admission he still had pain and a pelvic X-ray was requested (figure).

\author{
Department of \\ Medicine for the \\ Elderly, Wirral NHS \\ Trust Hospital, Arrowe \\ Park Road, Upton, \\ Wirral, Merseyside \\ L49 5PE, UK \\ R Morgan
}

Accepted 24 October 1997

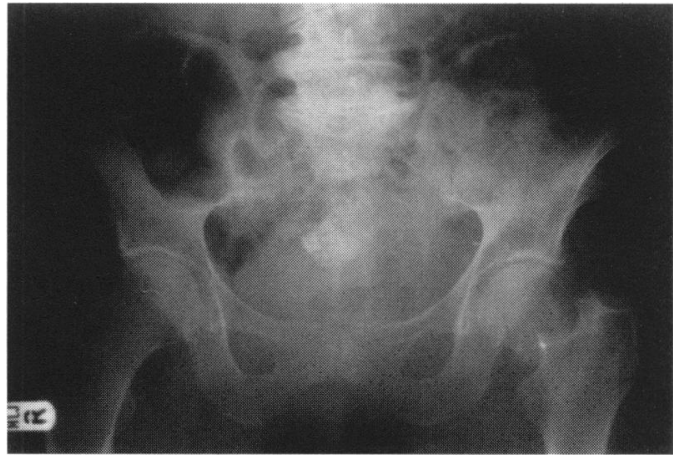

\section{Questions}

1 What is the diagnosis?

2 Why did he have 'knee' pain?

3 Why was there no obvious abnormality on inspection of both limbs?

4 What two factors predispose older people to this condition?

Figure Pelvic X-ray 